LMU-TPW 121-24

\title{
MULTIVALUED FIELDS ON THE COMPLEX PLANE AND BRAID GROUP STATISTICS
}

\author{
F. FERRARI \\ Sektion Physik der Universität München \\ Theresienstr. 37, 8000 München 2
}

Fed. Rep. Germany

\begin{abstract}
We study in this paper a theory of free anyons associated to free conformal field theories defined on Riemann surfaces with a discrete and nonabelian group of authomorphisms. The particles are exchanged according to a nonabelian statistics, in which the $R$-matrix satisfy a multiparametric generalization of the usual Yang-Baxter equations.
\end{abstract}

October 1992

1 Work supported by the Consiglio Nazionale Ricerche, P.le A. Moro 7, Roma, Italy 


\section{INTRODUCTION}

In two recent papers, [1] and [2], we have introduced a method for constructing conformal blocks with given monodromy properties, provided the monodromy properties can be related to those of an affine algebraic curve defined on the complex sphere $\mathbf{C P}_{1}$. The method was applied, as a first step, to the case in which the algebraic curves have a discrete group of symmetry $D_{m}$. Surely applications to more complicated curves are possible, but we do not have the proof that the method works in the most general case. Moreover, it is difficult to relate our conformal blocks to a conformal field theory at genus zero, but it is relatively simple to associate them with the theory of the $b-c$ systems on algebraic curves with $D_{m}$ group of symmetry. Also in the simple case discussed, the conformal blocks, builded in terms of free fields and twist fields [3], [4] that are explicitly known, exhibit nontrivial properties when two of the twist fields are exchanged. The twist fields turn out to be anions satisfying a nonabelian statistics. The purpose of this paper is to study the braid group statistics [5] of the twist fields.

\section{CONFORMAL FIELD THEORIES WITH NONABELIAN GROUP OF SYMMETRY}

In order to fix the notation, we briefly review the conformal field theories with nonabelian group of symmetries introduced in [2] and refs. [6], [1]. We begin considering the case in which the nonabelian group of symmetry is simply $D_{m}$. Further generalizations are possible as we will discuss in the next Section.

First of all let us introduce the $b-c$ systems of spin $\lambda(\lambda \in \mathbf{Z})$ on an algebraic curve $\Sigma_{g}$ :

$$
S=\int_{\Sigma_{g}} d^{2} z b \bar{\partial} c+\text { c.c. }
$$

$\Sigma_{g}$ is defined by the vanishing of the Weierstrass polynomial

$$
y^{2 m}-2 q(z) y^{m}+q^{2}(z)-p(z)=0
$$

where $q(z)$ and $p(z)$ are polynomials of degree $m r$ and $2 r^{\prime}$ respectively. The point $z=\infty$ is reached performing the substitution $z^{\prime}=1 / z$, so that the variables $z$ and $z^{\prime}$ represent a 
system of local coordinates describing the complex sphere $\mathbf{C P}_{1}$. The genus $g$ of the curve $\Sigma_{g}$ is given by the Riemann-Hurwitz formula:

$$
\begin{array}{ll}
2 g-2=2 m((m-1) r-2)+2 m r^{\prime} & m r \geq r^{\prime} \\
2 g-2=2 r^{\prime}(m-1)+2 m r^{\prime}-4 m & m r \leq r^{\prime}
\end{array}
$$

The solution of eq. (2.2) is a multivalued function (on $\left.\mathbf{C P}_{1}\right) y^{(l)}(z)$. The index $l=$ $0, \ldots, 2 m-1$ denotes the branches of $y(z)$, which are defined in the following way:

$$
\left\{\begin{array}{lll}
y^{(l)}(z)=e^{\frac{2 \pi i l}{m}} \sqrt[m]{q(z)+\sqrt{p(z)}} & 0 \leq l \leq m-1 \\
y^{(l)}(z)=e^{\frac{2 \pi i l}{m}} \sqrt[m]{q(z)-\sqrt{p(z)}} & m \leq l \leq 2 m-5
\end{array}\right.
$$

where $\epsilon^{m}=1$. The fact that the only two coordinates on $\Sigma_{g}$ are $z$ and $y(z)$, allows us to treat the $b-c$ systems as a multivalued field theory on the complex sphere. The function $y(z)$ has two different kinds of branch points. The branch points $\alpha_{i}, i=1, \ldots, N_{\alpha}=$ $\max \left(2 m r, 2 r^{\prime}\right)$ are the projections on $\mathbf{C P}_{1}$ of the zeros of $y(z)$. The branch points $\beta_{j}$, $j=1, \ldots, N_{\beta}=2 r^{\prime}$ represent instead the roots of the polynomial $p(z)$. For simplicity the integers $r$ and $r^{\prime}$ are chosen in such a way that $z=\infty$ is never a branch point. The local group of monodromy, describing the sequence in which the branches of the curve $y(z)$ are exchanged at the branch points, contains the nonabelian group $D_{m}$ as a subgroup.

If we put $\lambda>0$, a basis of meromorphic $\lambda$-differentials and $1-\lambda$-differentials in which all the elements have an independent behavior at the branch points, marked by the index $0 \leq k \leq 2 m-1$, is given by [2], [1]:

$$
\begin{aligned}
B_{k}^{(l)}(z) d z^{\lambda} & =\left(y^{(l)}(z)\right)^{m q_{k, \alpha_{i}}}(p(z))^{q_{k, \beta_{j}}} d z^{\lambda} \\
C_{k}^{(l)}(z) d z^{1-\lambda} & =\left(y^{(l)}(z)\right)^{-m q_{k, \alpha_{i}}}(p(z))^{-q_{k, \beta_{j}}} d z^{1-\lambda}
\end{aligned}
$$

where

$$
q_{k, \alpha_{i}}=\frac{[k]_{m}+\lambda(1-m)}{m} \quad\left[k_{m}\right]=[k+m]_{m}=k
$$

and

$$
\begin{aligned}
q_{k, \beta_{j}} & =-\frac{\lambda}{2} & k & =0, \ldots, m-1 \\
& =\frac{1-\lambda}{2} & k & =m, \ldots, 2 m-1
\end{aligned}
$$

In eq. (2.6) all the possible monodromy properties around the branch points that a meromorphic tensor can exhibit on $\Sigma_{g}$ are represented. Moreover any meromorphic tensor on 
$\Sigma_{g}$ can be expanded in terms of the elements of the basis (2.6), the coefficients of this expansion being rational singlevalued functions of $z$ [2]. As a consequence the $n$-point functions of the $b-c$ systems on an algebraic curve, explicitly computed using the method of fermionic construction [2], [7], are also decomposable in $n$-tensor products of the modes $B_{k}^{(l)}(z) d z^{\lambda}$ and $C_{k}^{(l)}(z) d z^{1-\lambda}$. In fact, the building blocks entering the $n$-point functions in the case $\lambda>1$ are the following tensors:

$$
K_{\lambda}^{\left(l l^{\prime}\right)}(z, w) d z^{\lambda} d w^{1-\lambda}=\frac{1}{2 m} \frac{d z^{\lambda} d w^{1-\lambda}}{z-w} \sum_{k=0}^{2 m-1} B_{k}^{(l)}(z) C_{k}^{\left(l^{\prime}\right)}(w)
$$

and the zero modes

$$
\Omega_{i_{k}, k}(z) d z^{\lambda}=z^{i-1} B_{k}(z) d z^{\lambda} \quad 1 \leq i_{k} \leq N_{b_{k}}
$$

$K_{\lambda}^{\left(l l^{\prime}\right)}(z, w) d z^{\lambda} d w^{1-\lambda}$ is characterized by a single pole in the variable $z$ when $z=w$ and $l=l^{\prime}$. In eq. (2.10) the index $i_{k}$ runs over all the zero modes $\Omega_{i_{k}, k}(z) d z^{\lambda}$ sharing the same monodromy properties of $B_{k}(z) d z^{\lambda}$. The number of these zero modes $N_{b_{k}}$ can be explicitly calculated in two ways: by explicit construction of the zero modes as in ref. [2] or from the residues of the ghost current $J^{(l)}(z)=: b(z) c(z)$ : as we will see below. The case $\lambda=1$ can be treated in an analogous way provided we replace $K_{\lambda}^{\left(l l^{\prime}\right)}(z, w) d z^{\lambda} d w^{1-\lambda}$ with the differential of the third kind:

$$
\omega_{a_{\left(l^{\prime}\right)} b_{\left(l^{\prime \prime}\right)}}^{(l)}(z) d z=K_{\lambda=1}^{\left(l l^{\prime}\right)}(z, a) d z-K_{\lambda=1}^{\left(l l^{\prime \prime}\right)}(z, b) d z
$$

This differential has two simple poles in $z=a$ and $z=b$ when $l=l^{\prime}$ and $l=l^{\prime \prime}$ respectively. Moreover, when $\lambda=1$, one has to consider also the zero mode in the $c$-fields, given by $C_{0}(z)=$ const. $d z^{0}$, so that $N_{c_{k}}=\delta_{k, 0} \delta_{1-\lambda, 0}$. This decomposition in $2 m k$-sectors, corresponding to the different elements of the basis (2.6), is also evident in the vacuum expectation values (vev) of the ghost current and of the energy momentum tensor $T(z)$. In fact, it is possible to pick up in these vev's the contributions $<T_{k}^{(l)}(z)>$ and $<J_{k}^{(l)}(z)>$ coming from the modes $B_{k}(z)$ that correspond to a given monodromy behavior at the branch points:

$$
<T^{(l)}(z)>=\frac{1}{2 m} \sum_{k=0}^{2 m-1}<T_{k}^{(l)}(z)>\quad<J^{(l)}(z)>=\frac{1}{2 m} \sum_{k=0}^{2 m-1}<J_{k}^{(l)}(z)>
$$


Since it will become useful for later purposes, we give the explicit expression of the vev $<J_{k}^{(l)}(z)>$ :

$$
<J_{k}^{(l)}(z)>d z=\frac{1}{2 m} \partial_{z} \log C_{k}^{(l)}(z) d z
$$

The vev $<T_{k}^{(l)}(z)>$ has been already derived in [2] and we do not report it here. It is possible to consider $\left\langle T_{k}^{(l)}(z)>\right.$ and $\left\langle J_{k}^{(l)}(z)>\right.$ as the vev's of the energy momentum and of the ghost current of the fields belonging to a given $k$-sector, distinguished by their monodromy behavior at the branch points. For example the particle content of the $k$-th sector is provided by the residues of $\left\langle J_{k}^{(l)}(z)>\right.$. In Section 5 of ref. [2] we showed that the total residue of $\left\langle J_{k}^{(l)}(z)>\right.$ at $z=\infty$ is $1-2 \lambda$. Moreover, in the case of a curve with nonabelian monodromy group, there are also the residues $q_{k, \alpha_{i}}(l)$ and $q_{k, \beta_{i}}(l)$ at the branch points, which are dependent on the branch $l$ of $J_{k}(z)$ in which they are computed:

$$
\begin{array}{rlrl}
q_{k, \alpha_{i}}(l) & =0 & 0 \leq l \leq m-1 \\
& =q_{k, \alpha_{i}} & & m \leq l \leq 2 m-1
\end{array}
$$

and

$$
q_{k, \beta_{j}}(l)=q_{k, \beta_{j}} \quad 0 \leq l \leq 2 m-1
$$

The charges $q_{k, \alpha_{i}}$ and $q_{k, \beta_{i}}$ were already given in eqs. (2.7) and (2.8). Summing the residues of $<J_{k}^{(l)}(z)>$ over all the possible branch points and over the branches $l=0, \ldots, 2 m-1$, we get the following equation determining the numbers of zero modes $N_{b_{k}}, N_{c_{k}}$ in a given $k$-sector:

$$
N_{b_{k}}-N_{c_{k}}=1-2 \lambda-\sum_{l=0}^{2 m-1} \sum_{i=1}^{N_{\alpha}} \frac{1}{2 m} q_{k, \alpha_{i}}(l)-\sum_{j=1}^{N_{\beta}} q_{k, \beta_{j}}
$$

The term $1-2 \lambda$ comes from the residue of the current $\left\langle J_{k}^{(l)}(z)>\right.$ at $z=\infty$ and it is the usual residue one would expect in the flat case. The residues at the branch points, proportional to $q_{k, \alpha_{i}}(l)$ and $q_{k, \beta_{j}}$, represent a nontrivial correction introduced by the global topology of the Riemann surface $\Sigma_{g}$. Finally, from eq. (2.16), it is clear that the total ghost charge in a given $k$-sector requires the introduction of $N_{b_{k}}-N_{c_{k}}$ zero modes, otherwise the amplitudes of the $b-c$ systems vanish identically. These $N_{b_{k}}+N_{c_{k}}$ zero modes are exactly those of eq. (2.10) having the same behavior at the branch points of a given element $B_{k}(z) d z^{\lambda}$ of the basis (2.6).

Finally, a study of the leading order poles of $\left\langle T_{k}(z)>\right.$ indicates that the effect of the branch points in the correlation functions can be simulated in a way that we will show 
later by the introduction of the socalled twist fields. These fields are conformal operators depending on the branch points and having fractional ghost charges. In the case in which the monodromy group of the algebraic curve is nonabelian, the dependence of the twist fields on the branch points becomes necessarily nonlocal.

One possibility to construct effectively a $b-c$ theory on $\Sigma_{g}$ in which the splitting of the fields is explicit, consists in expanding the fields in powers of $z$ and $y(z)$. This is the most general expansion allowed on an algebraic curve. Moreover, on an algebraic curve (2.2) there are only $2 m$ rationally independent functions $f_{k}(z)$, which are solution of a Riemann monodromy problem. The function $y(z)$ and its powers should be linear combinations of the $f_{k}(z)$ 's, the coefficients being rational functions of $z$ as explained above. Expanding also the rational functions in powers of $z$ and collecting all the terms in $f_{k}(z)$ together, $0 \leq k \leq 2 m-1$, we get the final form of the fields:

$$
\begin{gathered}
\sum_{k=0}^{2 m-1} \sum_{i=-\infty}^{\infty} z^{-i-\lambda} f_{k}(z) b_{k, i} \\
\sum_{k=0}^{2 m-1} \sum_{i=-\infty}^{\infty} z^{-i+\lambda-1} f_{k}(z) c_{k, i}
\end{gathered}
$$

Here $b_{k, i}$ and $c_{k, i}$ are arbitrary coefficients that in the quantum case become creation and annihilation operators of the fields propagating with the same monodromy properties of $f_{k}(z)$. This is the scheme used in [8] in order to construct an operator formalism for the $b-c$ systems on Riemann surfaces. Still there is an infinite number of possible solutions $f_{k}(z)$ of the Riemann monodromy problem, which are equivalent up to rational functions of $z$. The proper solutions for the $b-c$ systems are given in (2.6). Any other basis would produce an operator formalism in which the residues (2.14) and (2.15) of the currents $J_{k}(z)$ are shifted by integers. However this would be in contradiction with eqs. (2.14), (2.15) obtained computing explicitly the vev $\left\langle J_{k}(z)>\right.$ with the method of the fermionic construction [2].

\section{BRAID GROUP STATISTICS OF THE $b-c$ SYSTEMS}

Another possibility of realizing the theory of the $b-c$ systems on an algebraic curve in agreement with eq. (2.12) and (2.16) is based on bosonization [6], [1]. The details are described in ref. [2]. See also [9], [10] for a study of the $Z_{n}$ symmetric curves. Here we will 
mainly study the braid group statistics of the twist fields. First of all we associate to each $k$-sector with different monodromy properties at the branch points the couple of free $b-c$ fields $b_{k}(z) d z^{\lambda}$ and $c_{k}(z) d z^{1-\lambda}$. These fields take their values on the complex sphere and act on the vacua $\mid 0>_{k}, 0 \leq k \leq 2 m-1$. The fields belonging to different $k$-sectors do not interact. The presence of the branch points in the amplitudes is simulated by the twist fields $V_{k}^{\left(l_{i}\right)}\left(\alpha_{i}\right)$ and $V_{k}^{\left(l_{j}\right)}\left(\beta_{j}\right)$ for each sector $k$. These are primary fields as it is shown in [1] and [2]. The two point function of the fields $b_{k}$ and $c_{k}$ is defined as follows:

$$
G_{\lambda, k}^{\left(l l^{\prime}\right)}(z, w) d z^{\lambda} d w^{1-\lambda}=\frac{B_{k}^{(l)}(z) C_{k}^{\left(l^{\prime}\right)}(w)}{z-w} \prod_{s=1}^{N_{b_{k}}} \frac{\left(z-z_{k, s}\right)}{\left(w-z_{k, s}\right)} \prod_{s=1}^{N_{c_{k}}} \frac{\left(w-w_{k, s}\right)}{\left(z-w_{k, s}\right)} d z^{\lambda} d w^{1-\lambda}
$$

The meaning of eq. (3.1) as a two point function is due to the fact that this meromorphic tensor yields the vev's of the currents $J_{k}(z)$ and of the energy momentum tensors $T_{k}(z)$ discussed in the previous Section (see also [2]). Strictly speaking, however, this is not a true Green function on the algebraic curve because the structure of the poles is wrong. For example the pole in $z=w$ does not occur only when $l=l^{\prime}$ as it should be. However it is a Green function on the complex plane for the multivalued sector $k$.

It was shown in [2] that the explicit expression of eq. (3.1) in terms of twist fields and free $b-c$ fields is given by:

$$
G_{\lambda, k}^{\left(l l^{\prime}\right)}(z, w) d z^{\lambda} d w^{1-\lambda}=\frac{k<0\left|b_{k}(z) c_{k}(w) \prod_{s=1}^{N_{b_{k}}} b\left(z_{k, s}\right) \prod_{s^{\prime}=1}^{N_{c_{k}}} c\left(z_{k, s^{\prime}}\right) \prod_{i=1}^{N_{\alpha}} V_{k}\left(\alpha_{i}\right) \prod_{j=1}^{N_{\beta}} V_{k}\left(\beta_{j}\right)\right| 0>_{k}}{k<0\left|\prod_{s=1}^{N_{b_{k}}} b\left(z_{k, s}\right) \prod_{s^{\prime}=1}^{N_{c_{k}}} c\left(z_{k, s^{\prime}}\right) \prod_{i=1}^{N_{\alpha}} V_{k}\left(\alpha_{i}\right) \prod_{j=1}^{N_{\beta}} V_{k}\left(\beta_{j}\right)\right| 0>_{k}}
$$

Bosonizing the fields $b_{k}(z)$ and $c_{k}(z)$ with the rules:

$$
b_{k}(z)=e^{-i \varphi_{k}(z)} \quad c_{k}(z)=e^{i \varphi_{k}(z)} \quad<\varphi_{k}(z) \varphi_{k^{\prime}}\left(z^{\prime}\right)>=-\delta_{k k^{\prime}} \log \left(z-z^{\prime}\right)
$$

we get the following form of the twist fields:

$$
\begin{array}{r}
V_{k}^{(l)}\left(\alpha_{i}\right)=\exp \left[i \oint_{C_{\alpha_{i}}} d t<J_{k}^{(l)}(t)>\varphi_{k}(t)\right] \\
V_{k}^{(l)}\left(\beta_{j}\right)=\exp \left[i \oint_{C_{\beta_{j}}} d t<J_{k}^{(l)}(t)>\varphi_{k}(t)\right]=e^{-i q_{k, \beta_{j}} \varphi_{k}\left(\beta_{j}\right)}
\end{array}
$$


The vev of $J_{k}^{(l)}(z)$ is given explicitly in eq. (2.13) and $C_{\alpha_{i}}, C_{\beta_{j}}$ represent small paths surrounding the branch points $\alpha_{i}$ and $\beta_{j}$ respectively. The operators appearing in eqs. (3.4) and (3.5) satisfy a nonstandard statistics [2]:

$$
\begin{gathered}
V_{k}^{(l)}(\gamma) V_{k}^{\left(l^{\prime}\right)}\left(\gamma^{\prime}\right)= \\
\exp \left[-q_{k, \gamma} q_{k, \gamma^{\prime}} \oint_{C_{\gamma}} d s<J_{k}^{(l)}(s)>\oint_{C_{\gamma^{\prime}}} d s^{\prime}<J_{k}^{\left(l^{\prime}\right)}\left(s^{\prime}\right)>\log \left(s-s^{\prime}\right)\right] V_{k}^{\left(l^{\prime}\right)}\left(\gamma^{\prime}\right) V_{k}^{(l)}(\gamma)
\end{gathered}
$$

where $\gamma$ and $\gamma^{\prime}$ take their values on the set of branch points $\left\{\alpha_{i}, \beta_{j}\right\}$. In eq. (3.6), the dependence in the statistics of the twist fields on the branch points is reminiscent of the nonflat spacetime background on which the theory was originally defined. The $q$-parameter is a complicated convolution of three distributions. This is not in contrast with ref. [5], where it was shown that the dependence of the $q$-parameter on the spatial coordinates is excluded in the flat case due to the translational invariance of the local exchange relations. First of all we are here on a Riemann surface with a nontrivial geometry. Secondly, the algebra (3.6), defined on a local patch of the Riemann surface which is topologically equivalent to a complex plane with punctures at the branch points $\gamma$ and $\gamma^{\prime}$, becomes:

$$
V_{k}^{(l)}(\gamma) V_{k}^{\left(l^{\prime}\right)}\left(\gamma^{\prime}\right)=e^{i \pi q_{k, \gamma}(l) q_{k, \gamma^{\prime}}\left(l^{\prime}\right)} V_{k}^{\left(l^{\prime}\right)}\left(\gamma^{\prime}\right) V_{k}^{(l)}(\gamma)
$$

with $\gamma \neq \gamma^{\prime}$ and $l \neq l^{\prime}$. To derive eq. (3.7) we have simply evaluated the two line integrals occurring in eq. (3.6) using the following two equations, that express the coefficients appearing in eq. (2.2) in terms of the branch points:

$$
\begin{gathered}
q^{2}(z)-p(z)=\prod_{i=1}^{N_{\alpha}}\left(z-\alpha_{i}\right) \quad p(z)=\prod_{i=j}^{N_{\beta}}\left(z-\beta_{j}\right) \\
q(z)=\left(\prod_{i=1}^{N_{\alpha}}\left(z-\alpha_{i}\right)+\prod_{j=1}^{N_{\beta_{j}}}\left(z-\beta_{j}\right)\right)^{\frac{1}{2}}
\end{gathered}
$$

The remaining task is just a computation of residues. As a result, we see that the local algebra (3.7) has a $q$-parameter $\exp \left[i \pi q_{k, \gamma}(l) q_{k, \gamma^{\prime}}\left(l^{\prime}\right)\right]$ in which the dependence on the spatial coordinates is only appearing in the indices of the charges $q_{k, \gamma}(l)$ and $q_{k, \gamma^{\prime}}\left(l^{\prime}\right)$. 
At this point it is convenient to forget for the moment the index $k$ of the monodromy sector and to denote all the branch points with the symbol $\gamma_{i}, 1 \leq i \leq N_{\alpha}+N_{\beta}$, in such a way that:

$$
\begin{array}{lr}
\gamma_{i}=\alpha_{i} & 1 \leq i \leq N_{\alpha} \\
=\beta_{i-N_{\alpha}} & N_{\alpha}+1 \leq i \leq N_{\alpha}+N_{\beta}
\end{array}
$$

Moreover we group the the two indices $l_{i}$ and $\gamma_{i}$, describing the branch of $V_{k}^{\left(l_{i}\right)}\left(\gamma_{i}\right)$ and the branch point $\gamma_{i}$ simulated by the twist field respectively, in an unique index. The new composed indices will be denoted using the capital latin letters $I, J, K \ldots$ In this condensed notation we have for example that $V_{k}^{\left(l_{i}\right)}\left(\gamma_{i}\right) \equiv V^{I}, V_{k}^{\left(l_{j}\right)}\left(\gamma_{j}\right) \equiv V^{J}, 1 \leq i, j \leq N_{\alpha}+N_{\beta}$. Analogously $q_{k, \gamma_{i}}\left(l_{i}\right) \equiv q_{I}$ and so on. A sum over the index $I$, containing the indices $l_{i}$ and $\gamma_{i}, 1 \leq i \leq N_{\alpha}+N_{\beta}$, should be understood as a sum over the two indices $i$ and $l_{i}$. These two indices are totally independent. The subscript $i$ in $l_{i}$ is just a convenient notation when many twist fields are considered altogether, otherwise we could set $l_{i}=a, l_{j}=b$ and so on, $a, b=0, \ldots, 2 m-1$. The compact form of eq. (3.7) becomes:

$$
V^{I} V^{J}=e^{i \pi q_{I} q_{J}} V^{J} V^{I} \quad I \neq J
$$

In eq. (3.11) we have still not taken into account the radial ordering (or time ordering) that it is understood in eq. (3.2). As usual, two branch points are said to be radial ordered, i.e. $\gamma \prec \gamma^{\prime}$, iff $|\gamma|<\left|\gamma^{\prime}\right|$, the symbol || denoting the modulus of a complex number. Using this definition of time-ordering and eq. (3.11) we get:

$$
T\left(V^{I} V^{J}\right)=\left[e^{-i \pi q_{I} q_{J}} \theta(I-J)+e^{+i \pi q_{I} q_{J}} \theta(J-I)\right] T\left(V^{J} V^{I}\right)
$$

where $\theta(I-J) \equiv \theta\left(\left|\gamma_{i}\right|-\left|\gamma_{j}\right|\right)$ is the Heaviside theta function. The algebra (3.12) is trivially associative despite of the fact that the $q$-parameter $\exp \left(i \pi q_{I} q_{J}\right)$ depends also on the indices $I$ and $J$. In fact one can easily see that the following equation holds

$$
T\left(V^{I} V^{J} V^{M}\right)=\exp \left[i \pi\left(\epsilon(I, J) q_{I} q_{J}+\epsilon(I, M) q_{I} q_{M}+\epsilon(J, M) q_{J} q_{M}\right)\right] T\left(V^{M} V^{J} V^{I}\right)
$$

independently of the way in which the twist fields in the lhs are permuted in order to get the rhs. In (3.13) we have defined:

$$
\begin{aligned}
\epsilon(I, J) & =+1 \text { if } \gamma_{i} \prec \gamma_{j} \\
& =-1 \text { if } \gamma_{j} \prec \gamma_{i}
\end{aligned}
$$


The relations (3.12) resemble the equations defining the quantum hyperplane of [11], based on the quantum group $G L_{q}(N)$, where $q$ is a root of unity. We can interpret eqs. (3.2) and (3.12) in the following way. In the correlators of the $b-c$ systems on a Riemann surface with nonabelian monodromy group the dependence on the branch points becomes no longer local, but it is expressed through the twist fields (3.4) and (3.5). These operators replace the coordinates $\alpha_{i}$ and $\beta_{j}$ and can be considered as quantum coordinates. One is tempted therefore to treat the twist fields formally as coordinates of a complex hyperplane and to rewrite eq. (3.12) in a matricial form following refs. [11]:

$$
T\left(V^{I} V^{J}\right)=\sum_{M, N} Q_{M N}^{I J}\left(q_{I}, q_{J}\right) T\left(V^{M} V^{N}\right)
$$

where

$$
Q_{M N}^{I J}\left(q_{I}, q_{J}\right)=\exp \left(i \pi q_{I} q_{J}\right) \hat{R}_{m n}^{i j}\left[\exp \left(-i \pi q_{k, \gamma_{i}}\left(l_{i}\right) q_{k, \gamma_{j}}\left(l_{j}\right)\right)\right] \delta_{l_{n}}^{l_{i}} \delta_{l_{m}}^{l_{j}}
$$

Moreover $\hat{R}(q)$, with $q$ independent of the row indices $i, j$, satisfies the quantum YangBaxter equation:

$$
\hat{R}_{12} \hat{R}_{23} \hat{R}_{12}=\hat{R}_{23} \hat{R}_{12} \hat{R}_{23}
$$

and the sum over $M$ and $N$ in eq. (3.15) is intended as a sum over the small indices $m, n$ and $l_{m}, l_{n}$. The $\hat{R}_{m n}^{i j}$ component of $Q_{M N}^{I J}$ acts on the indices $i, j, m, n$ denoting the branch points $\gamma_{i}, \gamma_{j}, \gamma_{m}$ and $\gamma_{n}$, while the permutation matrix $\delta_{l_{n}}^{l_{i}} \delta_{l_{m}}^{l_{j}}$ acts on the branch indices $l_{i}, l_{j}, l_{m}$ and $l_{n}$. Explicitly eq. (3.15) reads:

$$
\begin{gathered}
T\left(V^{\left(l_{i}\right)}\left(\gamma_{i}\right) V^{\left(l_{j}\right)}\left(\gamma_{j}\right)\right)=\sum_{m, n=1}^{N_{\alpha}+N_{\beta}} \exp \left(i \pi q_{k, \alpha_{i}}\left(l_{i}\right) q_{k, \beta_{j}}\left(l_{j}\right)\right) \\
\hat{R}_{m n}^{i j}\left[\exp \left(-i \pi q_{k, \gamma_{i}}\left(l_{i}\right) q_{k, \gamma_{j}}\left(l_{j}\right)\right)\right] \sum_{i_{m}, i_{n}=0}^{2 m-1} \delta_{l_{n}}^{l_{i}} \delta_{l_{m}}^{l_{j}} T\left(V^{\left(l_{m}\right)}\left(\gamma_{m}\right) V^{\left(l_{n}\right)}\left(\gamma_{n}\right)\right)
\end{gathered}
$$

where we remember that the indices $l_{m}$ and $l_{m}$ are actually independent of $m$ and $n$. We can also generalize eq. (3.18) allowing for $q$-parameters which are not roots of unity. In this case $\exp \left(i \pi q_{I} q_{J}\right)$ can be substituted by any function $f\left(q_{I}, q_{J}\right)$ such that $f\left(q_{I}, q_{J}\right)=$ $f\left(q_{J}, q_{I}\right)$

The only difference between eq. (3.15) and the quantum hyperplane is that the $q$-parameter appearing in the matrix $Q_{M N}^{I J}$ depends now also on the row indices $I$ and $J$. 
The associativity property (3.13) requires that the matrix $Q$ of eq. (3.16) still satisfies a relation similar to the Yang-Baxter equations. Indeed it is easy to prove that:

$$
Q_{12}\left(q_{1}, q_{2}\right) Q_{23}\left(q_{2}, q_{3}\right) Q_{12}\left(q_{1}, q_{2}\right)=Q_{23}\left(q_{2}, q_{3}\right) Q_{12}\left(q_{1}, q_{2}\right) Q_{23}\left(q_{2}, q_{3}\right)
$$

This is not the usual Yang-Baxter equation, but a generalization of it that was firstly found in ref. [12]. In our case the form of eq. (3.19) is dictated by the fact that the twist fields obey the nonabelian statistics (3.11), which is a consequence of the nonabelian monodromy group of the algebraic curve (2.2). It is remarkable the fact that in refs. [13], [14]. Explicit solutions of eq. (3.19) were found from integrable models in which the spectral parameter is defined on algebraic curves of genus $g>1$. Multiparameter deformations of the quantum hyperplane such as in eq. (3.12) were also discussed in [15] and [16].

We conclude remembering that algebraic curves have many applications to knot theory [17]. Here we have expressed in terms of twist fields the braiding properties of the curve at the branch points. The situation in which the curve becomes degenerate, for example when two or more branch points coincide, is the most interesting for knot theory. However this situation is difficult to realize using the twist fields because the OPE's between two twist fields need some regularization when both of them are concentrated at the same branch point. 


\section{References}

[1] F. Ferrari, Phys. Lett 277B (1992), 423.

[2] F. Ferrari, Multivalued Fields on the Complex Plane and Conformal Field Theories, Preprint LMU-TPW 92-13.

[3] M. Sato, T. Miwa and M. Jimbo. Holonomic quantum fields (Kyoto U.P. Kyoto), part I; 14 (1978) p. 223; II: 15 (1979) p. 201; III: 15 (1979) p. 577; IV: 15 (1979) p. 871; $\mathrm{V} ; 16$ (1980) p. 531.

[4] L. Dixon, D. Friedan, E. Martinec, S. Shenker, Nucl. Phys. B282 (1987), 13; S. Hamidi, C. Vafa, Nucl. Phys. B279 (1987), 465; J. J. Atick, A. Sen, Nucl. Phys. B286 (1987), 189; L. Bonora, M. Matone, F. Toppan and K. Wu, Phys. Lett. 224B (1989) 115; Nucl. Phys. B334 (1990) 717; E. Guadagnini, M. Martellini, M. Mintchev, Jour. Math. Phys. 31 (1990), 1226.

[5] J. Fröhlich, Statistics of fields, The Yang-Baxter equation and the theory of knots and links, in: Nonperturbative quantum field theory, eds. G. t'Hooft et al. (Plenum, New York, 1988); Proceedings of the Gibbs Symposium, Yale University, D. G. Caldi, G. D. Mostow (eds.), (1990).

[6] F. Ferrari, Jour. Math. Phys. 32 (8), (1991) 2186.

[7] F. Ferrari, Int. Jour. Mod. Phys. A5 (1990), 2799.

[8] F.Ferrari, Operator Formalism on Algebraic Curves, Preprint MPI-Th/92-15.

[9] V. G. Knizhnik, Sov. Phys. Usp. 32(11) (1989) 945.

[10] M. A. Bershadsky and A. O. Radul, Int. Jour. Math. Phys. A2 (1987) 165.

[11] Yu. I. Manin, Comm. Math. Phys. 123 (1989), 163; J. Wess, B. Zumino, Nucl. Phys. B (Proc. Suppl.) 18B (1990), 302; W. Pusz, S. L. Woronowicz, Rep. Math. Phys. 27 (1989), 231.

[12] M. Jimbo, Int. Jour. Mod. Phys. A4 (1989), 3759.

[13] H. Au-Yang, B. M. Mc Coy, J. H. H. Perk, S. Tang, M. L. Yan, Phys. Lett. A123 (1987), 219

[14] R. J. Baxter, J. H. H. Perk, H. Au-Yang, it Phys. Lett. A128 (1988), 138

[15] D. Fairlie, C. Zachos, Phys. Lett 256B (1991), 43; C. Zachos, Proceedings of the XX-th International Conference on Differential Geometric Methods in Theoretical Physics, Eds. S. Catto and A. Rocha, Vol. II, World Scientific, (1992).

[16] A. Sudbery, J. Phys. A23 (1990), L697.

[17] E. Brieskorn, H. Knörrer, Plane Algebraic Curves, Birkhäuser Verlag (1986) 